\title{
Malignant Bowel Obstruction
}

National Cancer Institute

\section{Source}

National Cancer Institute. Malignant Bowel Obstruction. NCI Thesaurus. Code C141551.

Obstruction of the bowel by an advanced malignant tumor. It is a complication especially in patients with an advanced abdominal malignant tumor. 